\title{
Nanoneoadjuvant Therapy of Gastrointestinal Stromal Tumor (GIST)
}

\author{
Ronald P. DeMatteo, MD \\ Memorial Sloan-Kettering Cancer Center, New York, NY 10065
}

Although it is clear that imatinib mesylate is highly effective in metastatic gastrointestinal stromal tumor (GIST), its optimal perioperative use is undefined. McAuliffe and colleagues now report a provocative study of a brief preoperative imatinib therapy. ${ }^{1}$ Seventeen patients were randomized to receive 3,5 , or 7 days of $600 \mathrm{mg}$ imatinib, underwent surgery immediately thereafter, and then received 2 years of adjuvant imatinib therapy. Radiologic, histologic, and surgical outcome measures were analyzed.

By the authors' criteria, the response rate to ${ }^{18}$ FDG-PET $\left({ }^{18} \mathrm{~F}\right.$-fluoro-2-deoxy-D-glucose positron emission tomography) and dynamic computed tomography (CT) was approximately $70 \%$ after transient preoperative therapy. There was no mention of change in tumor density by CT, which has been the basis of the Choi criteria ${ }^{2}$ developed at their institution. Presumably it was unchanged. Pathologic analysis of 10 preoperative biopsy samples and their matched surgical specimens revealed that apoptosis (the primary endpoint of the trial) increased by a mean of $12 \%$. Early apoptosis has been shown previously in a mouse model of GIST after treatment with imatinib. ${ }^{3}$ Because early necrosis can be observed by TUNEL (terminal deoxynucleotidyl transferase dUTP nick-end labeling) staining as well, in the present study, it is uncertain whether apoptosis is the only mechanism responsible for tumor death. Interestingly, histologic cytoreduction was not apparent despite the high radiologic response rate. Whether patients without a radiologic response had evidence of apoptosis was not stated.

One item that will be of primary practical interest to surgeons is the fact that there was no difference in intraoperative blood loss, even though blood flow was reduced as measured by dynamic CT. This is surprising and

(c) Society of Surgical Oncology 2009

Published Online: 24 January 2009

R. P. DeMatteo, MD

e-mail: dematter@mskcc.org disappointing. It may just reflect patient selection, small sample size, and the constraints of using historical controls. The authors also conclude that compared with historical controls who did not receive neoadjuvant imatinib, there was no change in perioperative morbidity. However, the complication rate was $18 \%$ in the present series, versus $44 \%$ in controls $(P=.1)$. Similarly, postoperative complications did not appear to be altered in 45 patients with GIST treated with neoadjuvant imatinib on a Radiation Therapy Oncology Group phase II trial. ${ }^{4}$ Larger studies with proper controls are needed to identify whether neoadjuvant imatinib alters the rate of surgical complications.

Long-term outcome was assessed, although the analysis is limited by the mixture of patients with primary $(n=13)$ and metastatic $(n=6)$ disease. Recurrence-free survival exceeded $90 \%$ at 1 year, consistent with the preliminary data from the American College of Surgeons Oncology Group intergroup Z9001 adjuvant trial and metastatic GIST trials.

Although the current trial has primarily provided biologic and radiologic correlative information regarding neoadjuvant imatinib therapy in GIST, can the data be used in clinical practice? Is there a rationale to treat patients with resectable primary GIST for 1 week with neoadjuvant imatinib if intraoperative blood loss and perioperative morbidity are not affected? In patients with resectable primary GIST, there is a theoretical possibility that intraoperative tumor dissemination may be decreased by diminished tumor blood flow. This unproven benefit must be weighed against the rare occurrence of intraperitoneal tumor rupture induced by imatinib therapy. As more targeted drugs are developed, the other principal reason to use nanoneoadjuvant therapy for resectable GIST could be to select the most effective drug for adjuvant therapy in a given patient. However, until there is a strong clinical rationale, nanoneoadjuvant therapy for GIST may otherwise have a short life span. 


\section{REFERENCE}

1. McAuliffe JC, Hunt KK, Lazar AJ, et al. A randomized, phase II study of preoperative plus postoperative imatinib in GIST: evidence of rapid radiographic response and temporal induction of tumor cell apoptosis. Ann Surg Oncol.

2. Choi H, Charnsangavej C, Faria SC, et al. Correlation of computed tomography and positron emission tomography in patients with metastatic gastrointestinal stromal tumor treated at a single institution with imatinib mesylate: proposal of new computed tomography response criteria. J Clin Oncol. 2007;25:1753-9.

3. Rossi F, Ehlers I, Agosti V, et al. Oncogenic Kit signaling and therapeutic intervention in a mouse model of gastrointestinal stromal tumor. Proc Natl Acad Sci USA. 2006;103:12843-8.

4. Eisenberg BL, Harris $\mathrm{J}$, Blanke $\mathrm{CD}$, et al. Phase II trial of neoadjuvant/adjuvant imatinib mesylate (IM) for advanced primary and metastatic/recurrent operable gastrointestinal stromal tumor (GIST): early results of RTOG 0132/ACRIN 6665. J Surg Oncol. 2009;99:42-7. 